ROCZNIKI HUMANISTYCZNE

Tom LXVIII, zeszyt 8 - 2020

DOI: http://dx.doi.org/10.18290/rh.20688-10

\author{
XAVIER BLANCO \\ \& YAUHENIYA YAKUBOVICH
}

\title{
ÉLÉMENTS POUR UNE ANALYSE LEXICOLOGIQUE DES ENCYCLIQUES DE JEAN-PAUL II $^{*}$
}

\author{
ELEMENTS FOR A LEXICOLOGICAL ANALYSIS \\ OF THE ENCYCLICALS OF JOHN PAUL II
}

\section{Abstract}

This paper presents some examples of lexicological and syntactico-semantic analysis (using the linguistic engineering software NooJ 5.0) applied to the corpus of the fourteen Encyclicals of John Paul II. After a brief introduction, the first section $(\S 1)$ concerns the lexicometric aspect of the corpus. The second section $(\S 2)$ deals with the syntactic-semantic analysis and the identification of predicate-argument structures. The third section $(\S 3)$ presents some elements aimed at the study of collocations (restricted lexical combinatorics). The fourth and last section ( $§ 4)$ offers a start of French-Polish contrastive analysis.

Key words: argument schema; collocation; local grammar; lexical function; NooJ platform.

Prof. XAVier Blanco - Université autonome de Barcelone, Faculté des lettres ; adresse de correspondance: Bâtiment B. 08193 Bellaterra (Barcelone), Espagne ; courriel : xavier.blanco@ uab.cat. ORCID: https://orcid.org/0000-0001-8210-3668.

Dr Yauheniya Yakubovich - Université de Valence, Faculté de philologie, traduction et communication, Département de philologie française et italienne ; adresse de correspondance: Avda. Blasco Ibáñez, 32, 46010 Valencia, Espagne ; courriel : yauheniya.yakubovich@uv.es. ORCID: https://orcid.org/0000-0003-0630-8893.

Dr XAVIER Blanco - Uniwersytet Autonomiczny w Barcelonie, Wydział Literatury; adres do korespondencji: Bâtiment B. 08193 Bellaterra (Barcelone); e-mail: xavier.blanco@ uab.cat. ORCID: https://orcid.org/0000-0001-8210-3668.

Dr YauheniYa Yakubovich - Uniwersytet w Walencji, Wydział Filologii, Tłumaczeń i Komunikacji, Katedra Filologii Francuskiej i Włoskiej; adres do korespondencji: Avda. Blasco Ibáñez, 32, 46010 Valencia; e-mail: yauheniya.yakubovich@uv.es. ORCID: https:// orcid.org/ 0000-0003-0630-8893.

* Nous tenons à remercier ici chaleureusement Dorota Śliwa (Université Catholique de Lublin Jean-Paul II), ainsi que Dolors Català (Université autonome de Barcelone), qui ont relu le texte et ont formulé d'utiles observations. Nous remercions également les évaluateurs anonymes qui ont formulé un grand nombre de précisions importantes dont nous avons pu bénéficier. 


\section{INTRODUCTION}

Dans cet article, nous allons entamer une analyse lexicologique des Encycliques de Jean-Paul II en quatre volets. D'abord, nous mettrons en relief quelques caractéristiques lexicométriques de ces documents (identification et quantification de «mots-notion», $c f . \S 1)$. Ensuite, nous aborderons la combinatoire syntactico-sémantique de quelques-uns de ces mots-notion (reconnaissance de leurs structures prédicat-argument, $c f$. $\S 2$ ). Puis, nous ferons quelques commentaires à propos de leur combinatoire lexicale restreinte (collocations, $c f . \S 3$ ). Finalement, nous consacrerons un alinéa à la perspective contrastive français-polonais ( $c f . \S 4)$.

Il va sans dire que ce travail ne peut être qu'une toute première approche, très partielle, à ces textes extrêmement riches et profonds, qui ne prétend nullement à les décrire de façon exhaustive, encore moins à les interpréter. Il ne s'agit que d'une démonstration sommaire de l'application de quelques outils informatiques et de quelques principes de formalisation linguistique à l'analyse des discours mentionnés.

Précisons que Jean-Paul II est 1'auteur des quatorze Encycliques suivantes $^{1}$ (nous nous référons ici aux versions en langue française, traduites à partir du latin), qui s'étendent sur une période de vingt-quatre ans :

Ecclesia de Eucharistia, 17 avril 2003 ;

Fides et Ratio, 14 septembre 1998 ;

Ut Unum Sint, 25 mai 1995 ;

Evangelium vitce, 25 mars 1995 ;

Veritatis Splendor, 6 août 1993 ;

Centesimus Annus, $1^{\mathrm{er}}$ mai 1991 ;

Redemptoris Missio, 7 décembre 1990 ;

Sollicitudo Rei Socialis, 30 décembre 1987 ;

Redemptoris Mater, 25 mars 1987 ;

Dominum et Vivificantem, 18 mai 1986 ;

Slavorum Apostoli, 2 juin 1985 ;

Laborem Exercens, 14 septembre 1981 ;

Dives in Misericordia, 30 novembre 1980 ;

Redemptor Hominis, 4 mars 1979.

\footnotetext{
${ }^{1}$ Cf. « Jean-Paul II, Encycliques ». Le Saint-Siège, w2.vatican.va/content/john-paul-ii/fr/ encyclicals.index.html, consulté le 22.10. 2019.
} 


\section{ANALYSE LEXICOMÉTRIQUE}

Nous avons analysé les quatorze fichiers .pdf disponibles sur le site officiel du Saint-Siège à l'aide du logiciel d'ingénierie linguistique NooJ (version 5.0) (Silberztein, 2015) et des dictionnaires électroniques intégrés dans le module français. Du point de vue informatique, il s'agit d'un corpus qui contient 514359 tokens (de 26307 types différents) dont 23455 correspondent à des chiffres, 92509 correspondent à des caractères délimitateurs (espace, virgule, point-virgule...) et 398395 correspondent à des mots-formes (c'est-à-dire, à des séquences de lettres comprises entre deux caractères séparateurs). Le corpus comporte un total de 8412 unités textuelles délimitées par $I n$ (retour de chariot), correspondant grosso modo à des paragraphes. Les textes ont une taille moyenne de 25550 mots-formes (moyenne géométrique), Evangelium Vitae étant le plus long avec 49380 mots-formes et Slavorum Apostoli le plus court avec 10802 mots-formes.

Le corpus contient 36985 digrammes avec une fréquence égale ou supérieure à 2, c'est-à-dire, 36985 suites de deux mots-formes quelconques qui se répètent au moins une fois. Nous avons sélectionné, à l'intérieur de cette liste, les digrammes qui, tout en présentant une fréquence égale ou supérieure à 100, sont formés par un article défini (le, la, l', les) plus un substantif. Nous obtenons la liste suivante, contenant 59 digrammes (le chiffre entre parenthèses indique la fréquence absolue de chaque digramme dans le corpus) :

$\begin{array}{lr}\text { l'Église } & (1591) \\ \text { l'homme } & (1573) \\ \text { la vie } & (1164) \\ \text { l'Esprit } & (758) \\ \text { la vérité } & (588) \\ \text { la foi } & (567) \\ \text { le Christ } & (479) \\ \text { le monde } & (359) \\ \text { l'amour } & (331) \\ \text { l'histoire } & (315) \\ \text { les hommes } & (278) \\ \text { l'Evangile } & (267) \\ \text { la liberté } & (255) \\ \text { la personne } & (242) \\ \text { l'unité } & (221) \\ \text { la mission } & (214) \\ \text { le mystère } & (213) \\ \text { l'humanité } & (209)\end{array}$




$\begin{array}{ll}\text { la mort } & (204) \\ \text { la raison } & (198) \\ \text { la terre } & (198) \\ \text { la conscience } & (187) \\ \text { l'Eucharistie } & (172) \\ \text { le Concile } & (168) \\ \text { la société } & (162) \\ \text { la grâce } & (159) \\ \text { le Père } & (157) \\ \text { la lumière } & (155) \\ \text { le bien } & (154) \\ \text { l'esprit } & (146) \\ \text { la communion } & (145) \\ \text { la dignité } & (145) \\ \text { la Croix } & (144) \\ \text { l'activité } & (140) \\ \text { la loi } & (139) \\ \text { l'action } & (139) \\ \text { l'existence } & (135) \\ \text { la philosophie } & (133) \\ \text { la nature } & (133) \\ \text { le travail } & (132) \\ \text { le Seigneur } & (128) \\ \text { la justice } & (126) \\ \text { la miséricorde } & (124) \\ \text { le péché } & (124) \\ \text { la Loi } & (121) \\ \text { le sens } & (121) \\ \text { l'encyclique } & (120) \\ \text { l'œuvre } & (120) \\ \text { l'enseignement } & (114) \\ \text { le Fils } & (112) \\ \text { les paroles } & (111) \\ \text { la prière } & (111) \\ \text { la recherche } & (106) \\ \text { la création } & (106) \\ \text { l'expérience } & (105) \\ \text { le don } & (104) \\ \text { le fait } & (104) \\ \text { la culture } & (103) \\ \text { la doctrine } & (102) \\ & \\ & \end{array}$

La seule lecture de cette liste permet déjà de se faire une première idée du contenu et de la teneur de ces textes. Il est rare que la liste des digrammes 
les plus fréquents d'une collection de documents présente une telle cohérence du point de vue thématique, même quand il s'agit d'un corpus spécialisé. Cela indique que les encycliques ont bénéficié d'une planification textuelle et d'une rédaction extrêmement soignées. Il serait à noter également que seulement deux de ces syntagmes sont au pluriel (les hommes et les paroles).

\section{ANALYSE SYNTACTICO-SÉMANTIQUE}

Nous entendons par cooccurrence syntactico-sémantique le rapport qui existe entre les mots-clés cités et les prédicats appropriés qui les sélectionnent. Il ne nous est pas possible ici de commenter l'ensemble de la cooccurrence des mots-clés que nous avons mis en évidence. Ainsi donc, nous ferons uniquement quelques commentaires généraux à propos des substantifs les plus fréquents, de certains arguments qu'il sélectionnent et de certains prédicats qui s'y appliquent. Pour ce qui est de la méthodologie d'analyse linguistique, nous renvoyons le lecteur à Gross (Manuel d'analyse linguistique) pour ce qui est de l'analyse en schémas prédicat-argument(s) et à Silberztein pour l'élaboration et l'emploi de graphes en analyse syntactico-sémantique. Il est important de signaler que nous ne procédons pas à une discrimination précise des différents emplois des substantifs que nous commentons ici. Nos observations doivent donc être considérées comme approximatives.

Nous pouvons observer, par exemple, que seul un petit nombre de verbes prennent Église comme complément d'objet direct et qu'ils se concentrent dans l'encyclique Ecclesia de Eucharistia: édifier l'Église, modeler l'Église, raffermir l'Église, unir l'Église. Notons qu'ils contiennent les composantes sémantiques de construction ou de consolidation plus ou moins manifestes dans leur sens. Le cas de préserver l'Église est particulier. Nous trouvons, en effet, une répétition de cette combinaison à dix années de distance dans une phrase quasi identique, pour la bonne raison que Jean-Paul II se cite lui-même. Il s'agit d'un passage important que le Saint-Père a cru nécessaire de rappeler :

Slavorum Apostoli: La communion parfaite dans l'amour préserve l'Eglise de toute forme de particularisme et d'exclusivisme ethnique ou de préjugé racial, comme de toute arrogance nationaliste.

Ut Unum Sint : Ils mettaient en pratique « la parfaite communion dans l'amour [qui] préserve l'Eglise de toute forme de particularisme et d'exclusivisme ethnique ou de préjugé racial, comme de toute arrogance nationaliste ». 
Le substantif Église est sujet d'un nombre de verbes un peu plus large (une trentaine), où sont majoritaires les verbes de parole : déclarer, demander, désigner, exprimer ${ }^{2} .$. Notons qu'il s'agit ici de l'métonymique du substantif Église au sens de 'autorités de l'Église'.

Concernant les adjectifs, mis-à-part catholique, l'adjectif le plus fréquent qui se combine avec Église est universelle (notons que les deux adjectifs sont apparentés par le sens, du moins du point de vue étymologique), suivi de primitive.

Pour ce qui est du substantif Esprit, on en dénombre 758 occurrences dans le corpus, dont 447 correspondent au nom propre Esprit Saint (contre seulement 34 occurrences de Saint-Esprit). Comme on pourrait s'y attendre, l'encyclique Dominum et Vivificantem concentre la plupart des occurrences de cette locution nominale. La Figure 1 ci-dessous nous permet d'apprécier cette situation de façon visuelle (en termes de Standard Score ou Cote Z : 3,54 dans Dominum et Vivificantem par rapport à une fourchette d'entre 0,03 et $-0,41$ dans les autres encycliques).

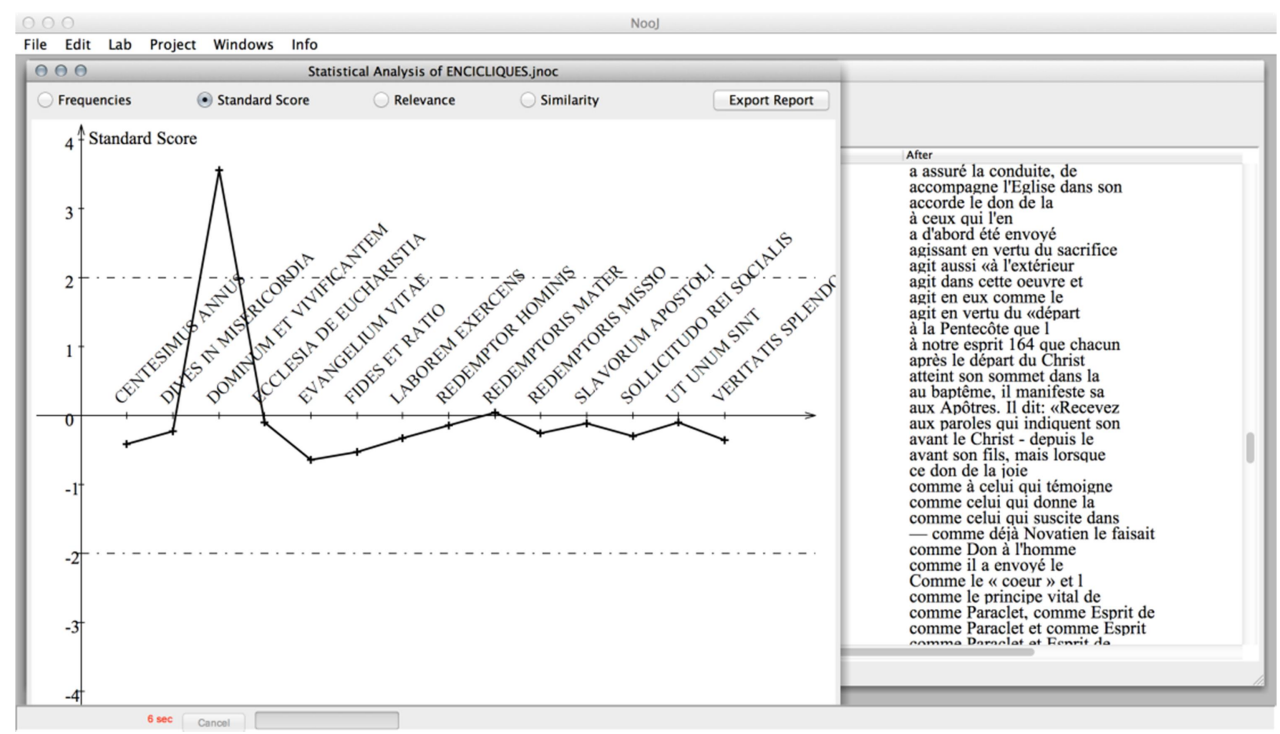

Figure 1: Occurrences de Esprit

\footnotetext{
${ }^{2}$ Bien entendu, la référence à la parole n'est pas exprimée de façon exclusive par des verbes, des constructions à verbe support sont également présentes : "Venite adoremus ». Telle est l'invitation que l'Église adresse à tous l'après-midi du Vendredi saint (Ecclesia de Eucharistia).
} 
Le nom propre Esprit Saint est sujet de verbes comme descendre, entrer ou venir, d'un côté, et agir de l'autre. En rapport avec le sens 'agir', nous trouvons toute une série de structures à verbe causatif (accorder le don de la conscience, faire comprendre que, faire entrer la lumière, offrir la possibilité de, répandre l'amour, etc.) qui précisent l'action de l'Esprit Saint et fonctionnent de facto comme des hyponymes d'agir.

Le digramme la vérité apparaît comme sujet d'un petit nombre de verbes prédicatifs et, en plus, le sémantisme de ces verbes n'est pas plein; il doit être précisé par les compléments d'objet direct (conduit la personne à, forme les consciences à, oriente l'action à). En revanche, ce syntagme est accompagné de nombreux adjectifs qui sont, en fait, des adjectivisations de verbes prenant vérité comme complément d'objet direct (en général, des verbes de parole) : la vérité exprimée, révélée, transmise... mais aussi d'autres types de verbes: la vérité atteinte, contenue, etc.

Le graphique suivant (Figure 2) illustre les occurrences de la vérité précédées d'une forme verbale quelconque. Il s'agit de la forme graphique générée par la sortie directe (sans post-édition d'aucune sorte) d'une requête de forme $\langle V\rangle$ la vérité lancée sur le logiciel NooJ. La netteté des résultats, qui est rare dans ce type de requêtes, somme toute assez générales, met en évidence, une fois de plus, que la rédaction des Encycliques a été méticuleuse. Mis à part être et faire (verbes à très large spectre sémantique) et les formes entre, contre et devant (qui correspondent à des prépositions dans le corpus, mais qui sont affichées dans cette figure à cause de l'homographie avec des formes appartenant au paradigme des verbes entrer, contrer et devoir), le graphique nous montre quels sont les verbes prenant la vérité comme complément d'objet direct dans le corpus étudié.

Nous pouvons observer que connaître et chercher sont les verbes qui se combinent le plus souvent avec la vérité et ceux qui sont aussi les mieux distribués dans les différentes encycliques. La suite connaître la vérité apparaît dans sept d'entre elles: Centesimus Annus, Dominum et Vivificantem, Fides et Ratio, Redemptoris Hominis, Redemptoris Mater, Redemptoris Missio et Veritatis Splendor. La suite chercher la vérité dans quatre : Centesimus Annus, Fides et Ratio, Redemptoris Missio et Veritatis Splendor. Soulignons que les différentes formes flexionnelles d'un verbe donné n'ont pas été regroupées dans le graphique ci-dessus (le logiciel permet cependant de le faire si nécessaire). 


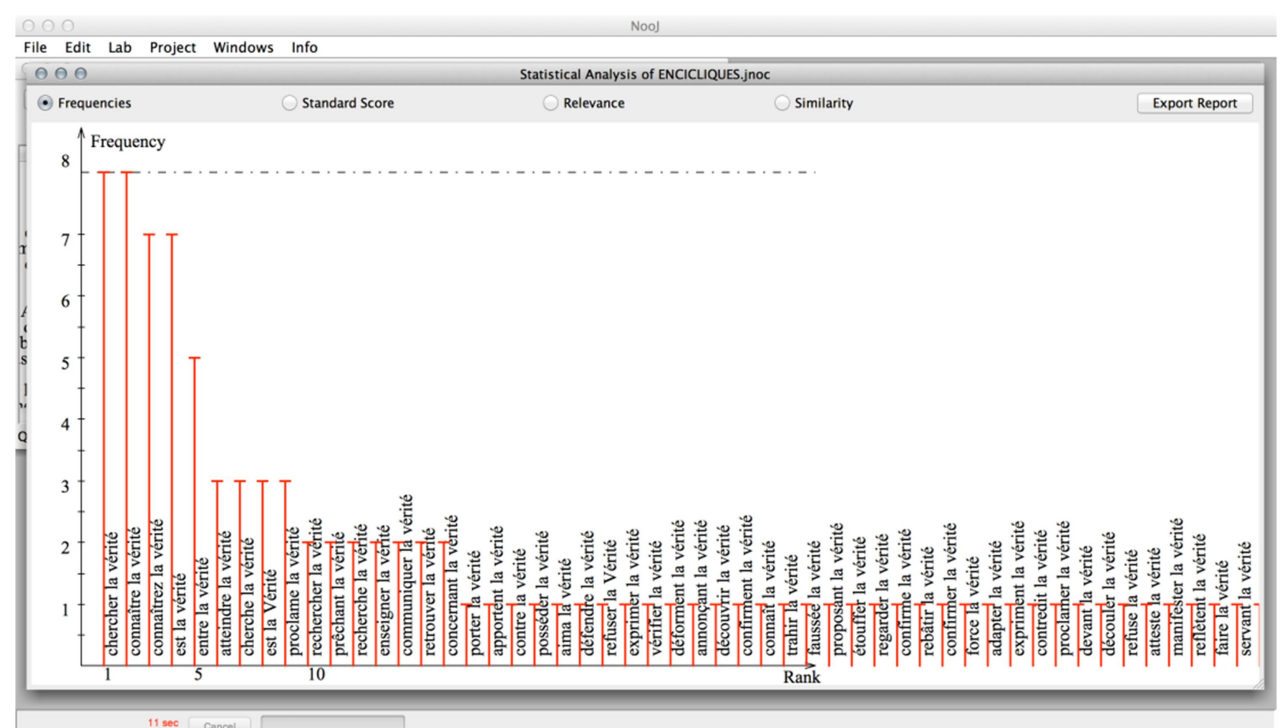

Figure 2 : Vérité suivie de forme verbale

Afin de faire varier notre point de vue, nous examinerons maintenant la cooccurrence nominale, et non pas verbale, du syntagme la foi. Il figure, avec un autre nom prédicatif ${ }^{3}$ et dans une structure $\mathrm{N}_{1}$ Prép $\mathrm{N}_{2}$ (nous ne retenons que les noms présentant un nombre d'occurrences élevé) dans : le contenu de la foi, la diffusion de la foi (expansion et propagation sont moins fréquentes), les données de la foi, les exigences de la foi, l'intelligence de la foi, la maturité de la foi, l'obéissance de la foi (souvent par rapport à Marie, mais aussi en référence à $\mathrm{Rm} 16,26$; Rm 1,6 et 2 Co 10 5-6), le pèlerinage de la foi, l'unité de la foi, les vérités de la foi... En général, la préposition est de, mais nous avons aussi : l'appel à la foi, la participation à la foi, etc. Il serait à noter, également, que le syntagme prépositionnel à la lumière de la foi présente une haute fréquence (12 occurrences, à savoir : 8 dans Fides et Ratio, 2 dans Sollicitudo Rei Sociales et 2 dans Veritatis Splendor).

La cooccurrence syntactico-sémantique d'un mot-concept donné dans le corpus peut être modélisée et représentée moyennant un graphe qui rend compte d'un transducteur à états finis. Un exemple illustrant la cooccurrence du mot-notion l'unité ('unité de l'Église') pourrait être celui de la Figure 3 ci-dessous.

\footnotetext{
${ }^{3}$ Insistons sur le fait que nous ne prenons à chaque fois qu'une des nombreuses possibilités d'analyse. Par exemple, si nous considérions également les $\mathrm{N}_{1}$ de type humain, nous trouverions : hérauts de la foi, martyrs de la foi, témoins de la foi, etc.
} 


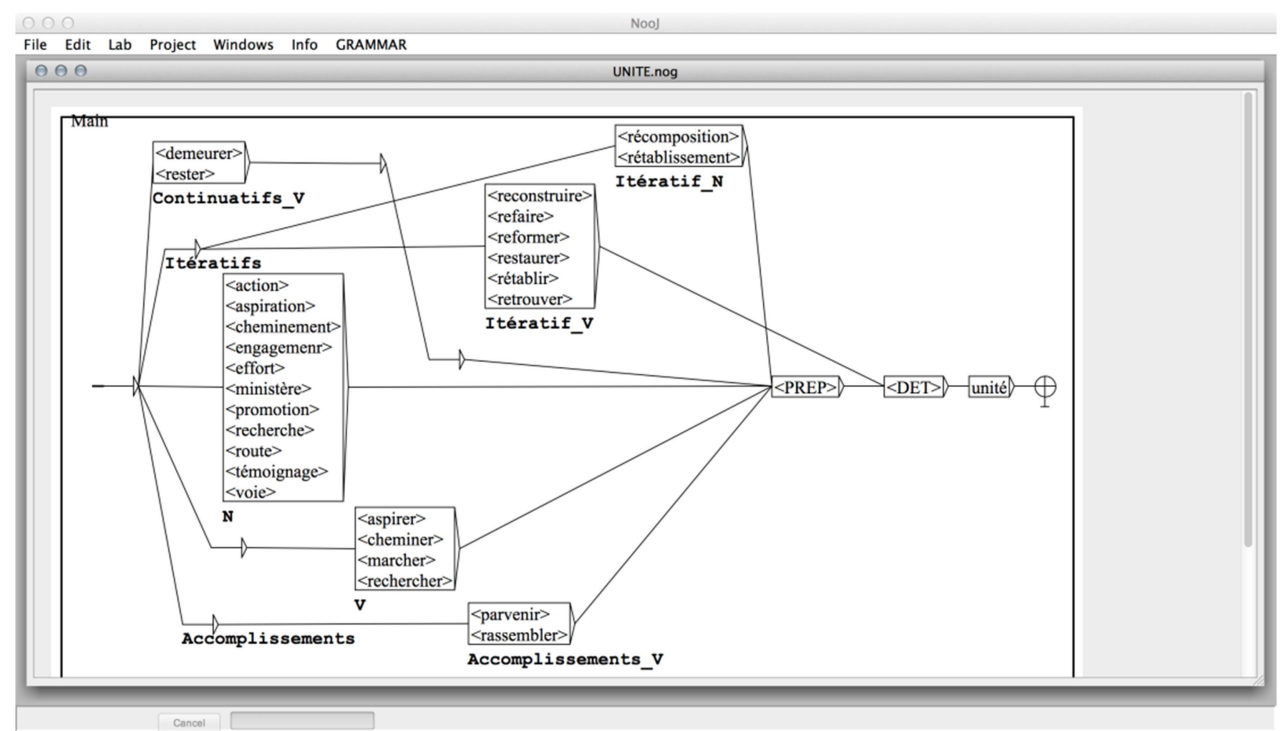

Figure 3 : Cooccurrence d'unité

Bien entendu, une grammaire locale ${ }^{4}$ n'épuise pas les possibilités d'expression liées à une notion donnée. La figure ci-dessus montre, de façon simplifiée et stylisée (afin que l'image reste interprétable), une certaine quantité des contextes de l'unité dans le corpus. La prise en compte de suites comme effort commun vers la plénitude dans l'unité ou rassembler dans l'unité des enfants de Dieu dispersés exigeraient cependant l'ajout de nouveaux noyaux (insertion d'adjectif après le substantif, insertion d'une structure $\mathrm{N}_{1}$ de $\mathrm{N}_{2}$ où l'unité correspondrait au $\mathrm{N}_{2}$, etc.).

\section{ANALYSE COLLOCATIONNELLE}

Il serait à noter que les encycliques de Jean-Paul II semblent éviter tout cliché superflu et, par conséquent, ne multiplient pas les occurrences des collocations standard. L'expression est maîtrisée et l'adjectivation riche, mais pondérée, fuyant en tout moment le ton emphatique.

Il existe une soixantaine de collocations standard. Il est impératif de faire un choix. Nous examinerons ici uniquement les collocations mélioratives

\footnotetext{
${ }^{4}$ Une grammaire locale, au sens linguistique du terme, généralise la notion de schéma d'arguments en présentant toute une série de phrases potentielles appropriées à un prédicat. Sur cette notion, on peut consulter, par exemple Gross ( «Une grammaire locale »).
} 
(qui expriment le sens 'bon') présentes dans l'encyclique Ut Unum Sint. Après les collocations intensives (qui expriment le sens 'très', 'intense') ${ }^{5}$, elles sont probablement les plus nombreuses, aussi bien en langue qu'en discours. Dans le cadre de ce survol rapide, il ne nous sera pas toujours possible de distinguer soigneusement entre collocation intensive (Magn), méliorative (Bon), vériconditionnelle (Ver) ou superlative $(\mathbf{C u l m})^{6}$, car certains cas exigeraient une analyse sémantique minutieuse en vue d'une attribution exacte.

Nous trouvons, comme collocatifs de type mélioratif (ou présentant un sens proche), les adjectifs, les adverbes et les syntagmes prépositionnels suivants (par ordre alphabétique) :

accompli, admirable, authentique, avec conviction, avec douceur, avec ferveur, avec patience, avec puissance, avec raison, avec sagesse, avec satisfaction, avec sobriété, avec vigueur, béni, central, clairement, commun, concret, confiant, convenablement, courageux, de façon claire, de façon vivante, de manière éloquente, désintéressé, digne, efficacement, éloquent, enthousiasmant, essentiel, expressif, facile, fécond, ferme, fidèle, fondamental, fraternel, généreux, historique, humblement, important, intime, juste, légitime, libre, librement, loyal, lucide, luxuriant, naturel, opportunément, ouvert, pacifique, parfait, pleinement, positif, précieux, profond, réel, sacré, sain, saint, salutaire, salvifique, sanctifiant, sérieux, significatif, sincère, sincèrement, solennel, solidaire, tangible, unique, utile, vénéré, véritable, visible, vrai.

Quelques-unes des combinaisons dans lesquelles peuvent entrer ces lexies correspondent bel et bien à des collocations mélioratives stricto sensu (le collocatif y est sélectionné lexicalement en fonction du collocateur, ou base de la collocation, afin d'exprimer le sens 'bon, convenable'): offrande généreuse, fruits positifs, volonté sincère, démarche utile, citer opportunément, déclarer clairement, présenter de façon claire, exprimer de façon vivante, agir efficacement, travail important, fruit précieux, affection sincère. Dans certains cas, l'adjectif mélioratif présente une combinatoire assez variée. Ainsi, courageux entre dans les combinaisons ${ }^{7}$ : témoignage courageux, projets courageux, travail courageux, courageux cheminement.

\footnotetext{
${ }^{5}$ Sont des collocations intensives (présentes dans l'encyclique en question) des suites comme : désir ardent, espérance inébranlable, devoir impérieux, insigne importance, se rencontrer assidûment, grave difficulté, engagement profond, adhérer fermement, importance primordiale, associer inséparablement, insondable richesse, dimension vaste, offrande totale, ferme conviction.

${ }^{6}$ Nous précisons, en caractères gras, les fonctions lexicales qui permettent de modéliser ces différents types de collocations dans le cadre de la Lexicologie Explicative et Combinatoire (Mel'čuk, « Collocations »; Mel'čuk et Polguère).

${ }^{7}$ Rappelons que les exemples ici cités ne concernent que l'encyclique Ut Unum Sint. Le dépouillement systématique des encycliques restantes nous permettrait de mettre en évidence de
} 
D'autres suites qui pourraient, en apparence, correspondre à des collocations sont, en fait, des locutions nominales, et vice-versa. Ainsi, par exemple, pleine communion n'est plus à considérer comme une collocation intensive ni méliorative, mais comme une locution qui correspond à un concept bien précis en tant qu'étape avancée d'un processus de convergence des Églises. La suite parfaite communion, par contre, peut-être considérée encore comme une collocation (qui admet, sous la plume de Jean-Paul II, le préfixe négatif : la communion encore imparfaite de nos communautés...). Un exemple a contrario serait la suite pleine conscience, qui est, à tous les effets, une collocation intensive dans le texte de l'encyclique Ut Unum Sint, mais qui correspondrait à une locution nominale dans tout autre texte où elle serait employée en tant que traduction de Mindfulness.

Dans de nombreux cas, l'association lexicale n'a pas le caractère strict d'une collocation, car l'adjectif ou l'adverbe à sens mélioratif présentent une certaine liberté combinatoire. Dans certaines suites N Adj ou V Adv on est face à des cas intermédiaires entre sélection lexicale de la part du $\mathrm{N}$ ou du V (relation collocateur-collocatif) et sélection sémantique de la part de l'Adj ou de l'Adv (relation prédicat-argument).

Un exemple d'adjectif qui garde un spectre combinatoire assez large serait fraternel, qu'on trouve appliqué à charité, communion, concorde, coordination, dialogue, émulation, générosité, influence, koinônia 'communion', respect, soutien.., tout en pouvant être considéré comme collocationel dans certaines suites telles que relations fraternelles. Nous présentons ci-dessous la concordance de fraternel et de ses formes fléchies ( $<$ fraternel $>$ ) extraite de l'encyclique Et Unum Sint :

exclusions qui blessent la charité fraternelle, de certains refus de pardonner service, celle d'une générosité fraternelle envers les autres ». Prier pour incitera tous, dans une émulation fraternelle, à une connaissance plus profonde un esprit sincère de charité fraternelle, de respect des exigences de commune est riche de communion fraternelle, mais elle est aussi une

nombreuses autres cooccurrences. Avec courageux, nous avons, par exemple, recherche courageuse, qui est absente de l'encyclique mentionnée, mais présente dans Fides et Ratio et dans Slavorum Apostoli. Il serait nécessaire, ensuite, de procéder à l'analyse de chacun des contextes. Nous pourrions observer ainsi que la collocation méliorative recherche courageuse concerne deux sens différents de recherche : le sens 'Activités intellectuelles, travaux ayant pour objet la découverte, l'invention, la progression des connaissances nouvelles' (Trésor de la langue française informatisé, s.v. recherche A.2) dans Fides et Ratio et le sens 'Action de chercher (pour trouver) quelque chose; efforts entrepris.' (Trésor de la langue française informatisé, s.v. recherche B.2) dans Slavorum Apostoli. 
sont convertis à une charité fraternelle qui englobe tous les disciples en vue d'une coordination fraternelle. Par ailleurs, notre participation mutuelle et de charité les relations fraternelles qui doivent être en honneur non seulement un vrai respect fraternel entre orthodoxes et catholiques vivant catholique a renoué des relations fraternelles avec les Eglises anciennes joie. La reprise des relations fraternelles avec les Eglises anciennes recherche théologique et du dialogue fraternel. Et il y a plus attitude dictée par la charité fraternelle et empreinte d'une foi est la base de relations fraternelles bien différentes d'une entente Les liens de la koinônia fraternelle se nouent devant Dieu et efforce de susciter un soutien fraternel mutuel par lequel les Communautés en bonne place cette influence fraternelle réciproque. Au point où nous étaient unis par la communion fraternelle dans la foi et la sur ce sujet un dialogue fraternel et patient, dans lequel nous paix, c'est la concorde fraternelle, c'est le peuple rassemblé

Abondent, dans les Encycliques de Jean-Paul II, les adjectifs qui ont une composante 'vrai' dans des combinatoires de type mélioratif. C'est le cas, en particulier, de authentique, sincère, véritable et vrai. Voici les concordances de authentique et de sincère, toujours dans l'encyclique mentionnée :

pas d'œcuménisme au sens authentique du terme sans conversion intérieure de l'unité », " une expression authentique des liens par lesquels les condition essentielle de toute recherche authentique de l'unité, naît de Pape Paul VI, en un authentique « dialogue du salut». Le dialogue plus profonde d'une recherche authentique de la pleine communion entre mystère du Christ. L'œcuménisme authentique est une grâce de vérité fassent preuve d'un héroïsme authentique dans leur décisions. A ce la foi catholique, le magistère authentique tient une place particulière pour sauront-ils accueillir le message authentique? Ne penseront-ils pas que notre engagement soit toujours plus authentique. Et si nous devions nous

ainsi que par la volonté sincère de se pardonner mutuellement reconnaître ensemble, avec une objectivité sincère et totale, les erreurs commises vie, manifestent un zèle religieux sincère, croient avec amour en Dieu demander la grâce d'une sincère abnégation, celle de l'humilité soient abordées dans un esprit sincère de charité fraternelle, de respect à Rome avec une affection sincère et avec la solennité qui est fait avec une volonté sincère d'adhérer à la Parole la justice et une charité sincère envers le prochain ». Ces remarques il y a une volonté sincère de suivre le Christ, l'Esprit sait souvent grâce à la recherche commune, sincère et désintéressée de la vérité 


\section{ANALYSE CONTRASTIVE FRANÇAIS-POLONAIS}

Avant de passer à l'analyse des Encycliques en polonais, il nous semble important de mettre en relief des différences typologiques entre le français, une langue romane fortement germanisée, et le polonais, une langue slave occidentale.

Le polonais ne possède pas d'articles et, par conséquent, n'a pour déterminants que les pronoms, souvent absents. Cela rapproche, selon son synthétisme uniquement, le polonais au latin et fait qu'un texte polonais contienne, en général, moins de signes linguistiques (tokens) que son équivalent français. Potentiellement, le texte polonais aurait également moins de prépositions que le français puisque les noms et les adjectifs se déclinent. Grâce au système des cas, l'ordre des mots est moins rigide en polonais qu'en français.

Quant à notre corpus, le texte polonais de la totalité des Encycliques comprend 412669 tokens (contre 514359 en français) et 32188 digrammes (contre 36985 pour le corpus français) avec une fréquence égale ou supérieure à 2 . $\mathrm{Vu}$ le caractère plus synthétique du polonais, les digrammes français Art_Déf N sont parfois remplacés, en polonais, par des substantifs avec d'autres types de déterminants, comme les pronoms. Le plus souvent, toutefois, on les retrouve sous forme d'unigrammes, i.e. des substantifs. Comparons, dans le fragment déjà cité de Slavorum Apostoli :

FR : La communion parfaite dans l'amour préserve l'Eglise de toute forme de particularisme et d'exclusivisme ethnique ou de préjugé racial, comme de toute arrogance nationaliste.

PL : Doskonała wspólnota milości chroni Kościół od wszelkiego partykularyzmu. Wyłączności etnicznej, uprzedzeń rasowych czy narodowej pychy.

Pour la même raison (degré différent de synthétisme), aux deux formes du substantif français Église (singulier et pluriel) correspondent, dans le corpus polonais, douze mots-formes possibles de Kościót (singulier et pluriel des six cas). Selon les fréquences dans la totalité des Encycliques, nous avons :

Kościoła,TOKEN+Freq=916

Kościół,TOKEN+Freq=710

Kościele, TOKEN + Freq $=381$

Kościołów, TOKEN+Freq $=135$

Kościołem,TOKEN+Freq $=86$

Kościoły,TOKEN+Freq $=59$

Kościołowi, TOKEN+Freq $=57$

Kościołami,TOKEN+Freq=39 


\section{Kościołach,TOKEN+Freq $=25$ \\ Kościołom,TOKEN + Freq $=9$ \\ KOŚCIOŁEM,TOKEN+Freq $=5$ \\ kościele,TOKEN+Freq $=4$ \\ KOŚCIOŁA,TOKEN+Freq $=4$ \\ kościołów,TOKEN+Freq=3 \\ KOŚCIOŁÓW,TOKEN+Freq=3 \\ kościół,TOKEN+Freq=3 \\ kościoła,TOKEN+Freq $=2$ \\ kościołach,TOKEN+Freq $=2$ \\ KOŚCIÓŁ,TOKEN+Freq=1 \\ Kościóła,TOKEN+Freq=1}

Pour rendre le sens syntaxique exprimé par un cas polonais, on aurait besoin, en français, d'une préposition : de l'Église, à l'Église, etc.

En ce qui concerne les cooccurrences lexico-sémantiques dans les textes des Encycliques, mentionnons les énoncés où Duch (Esprit) où Duch (Święty), Esprit (Saint), remplit la fonction de sujet. Nous avons détecté 52 cas où le sujet précède le prédicat :

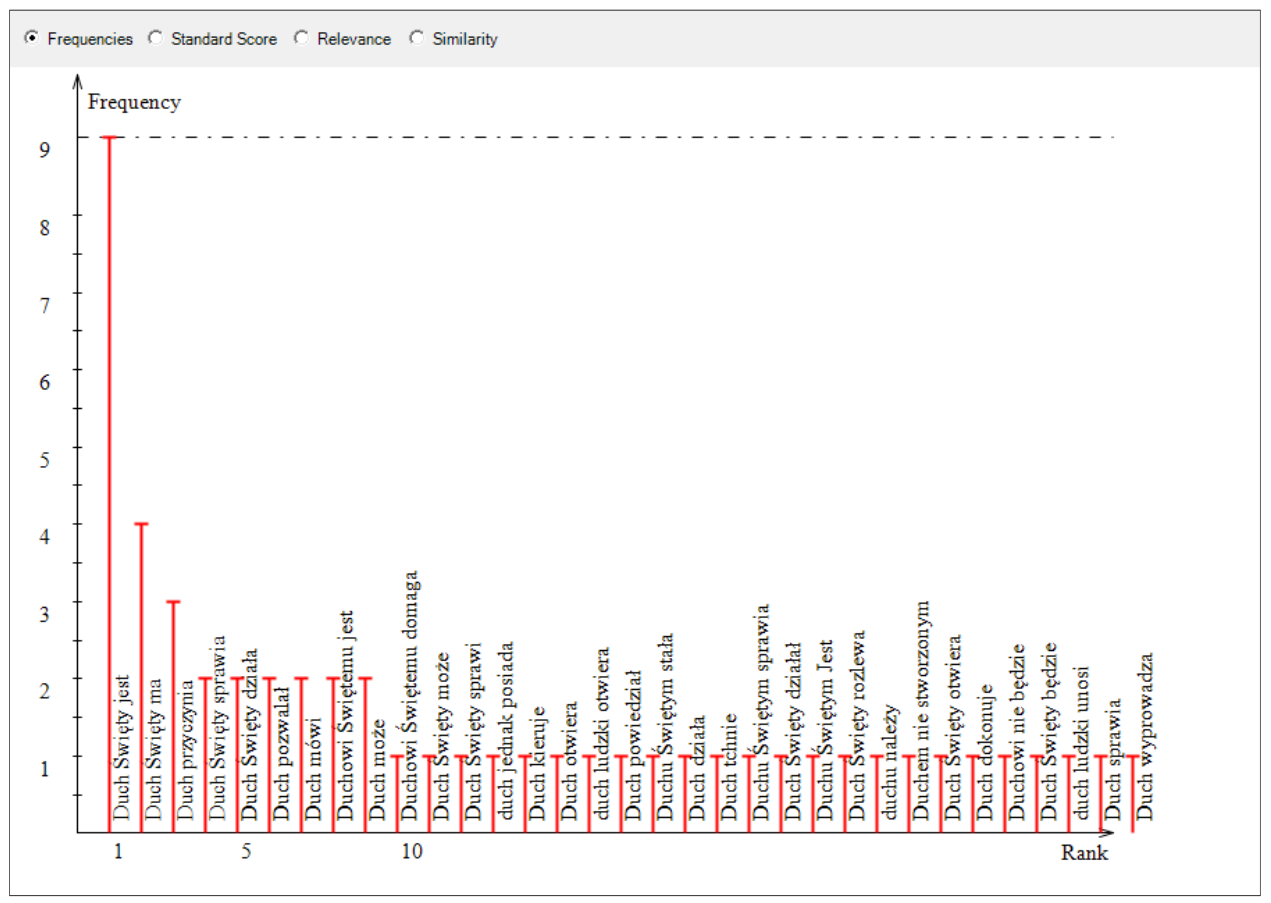

Figure 4 : Duch comme premier actant sémantique 
Il faut tenir compte du fait que, en polonais, le sujet peut suivre le prédicat. Nous avons repéré 18 occurrences avec Duch en post-position. Aussi bien dans la version française des Encycliques que dans les versions polonaises, Duch est souvent sujet du verbe działać avec le sens 'agir' et d'un prédicat de sens causatif, comme dans sprawiać ou przyczyniać się. Il se combine également avec des verbes présentant d'autres sens, e.g. pozwalać ('permettre'), mowić ('parler'), panować ('régner'), etc.

Nous avons rencontré très peu d'occurrences où Duch remplit la fonction de complément d'objet direct (substantif en accusatif : biernik) : otrzymać Ducha Świętego (recevoir l'Esprit Saint), powierzać ducha (remettre l'esprit) ${ }^{8}$, posiadać ducha (posséder l'Esprit), mieć Ducha (avoir l'Esprit) sont les seules formules de ce genre détectées dans le corpus.

Quant aux modifieurs du substantif en question, nous trouvons 282 occurrences de la structure Duch Adj, comme Duch Swiety (l'Esprit Saint), duch ludzki (l'esprit humain) ou Duch Boży (l'Esprit de Dieu), ainsi que 29 cas de la structure inverse (Adj Duch), avec des occurrences comme nowy duch (un esprit nouveau), jeden duch (l'unique Esprit), Boży Duch (l'Esprit de Dieu) ou inny duch (un autre esprit). Notons aussi la reprise anaphorique de l'adjectif Saint ou du groupe de Dieu par le déterminant démonstratif (ten en polonais et ce en français) et par l'adverbe (sam-même) composant un déterminant complexe, comme par exemple ten sam Duch (ce même Esprit) dans Veritatis Splendor.

On trouve quelques occurrences ( 25 dans le corpus) du groupe nominal

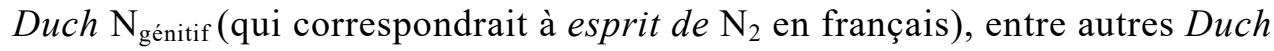
Chrystusa (l'esprit du Christ), duch Boga Stwórcy (l'esprit de Dieu Créateur), etc. La plupart de ces occurrences de Duch ou duch réfèrent à Dieu, mais 9 d'entre elles réfèrent à une valeur, comme par exemple $d u c h$ pokoju i przebaczenia (un esprit de paix et de pardon).

\footnotetext{
${ }^{8}$ Remettre l'esprit, dans le texte de l'encyclique Evangelium Vitce, correspond à l'Évangile selon Saint Luc (23:46), où Jésus exclame : «Ojcze, w Twoje ręce powierzam ducha mojego ».
} 


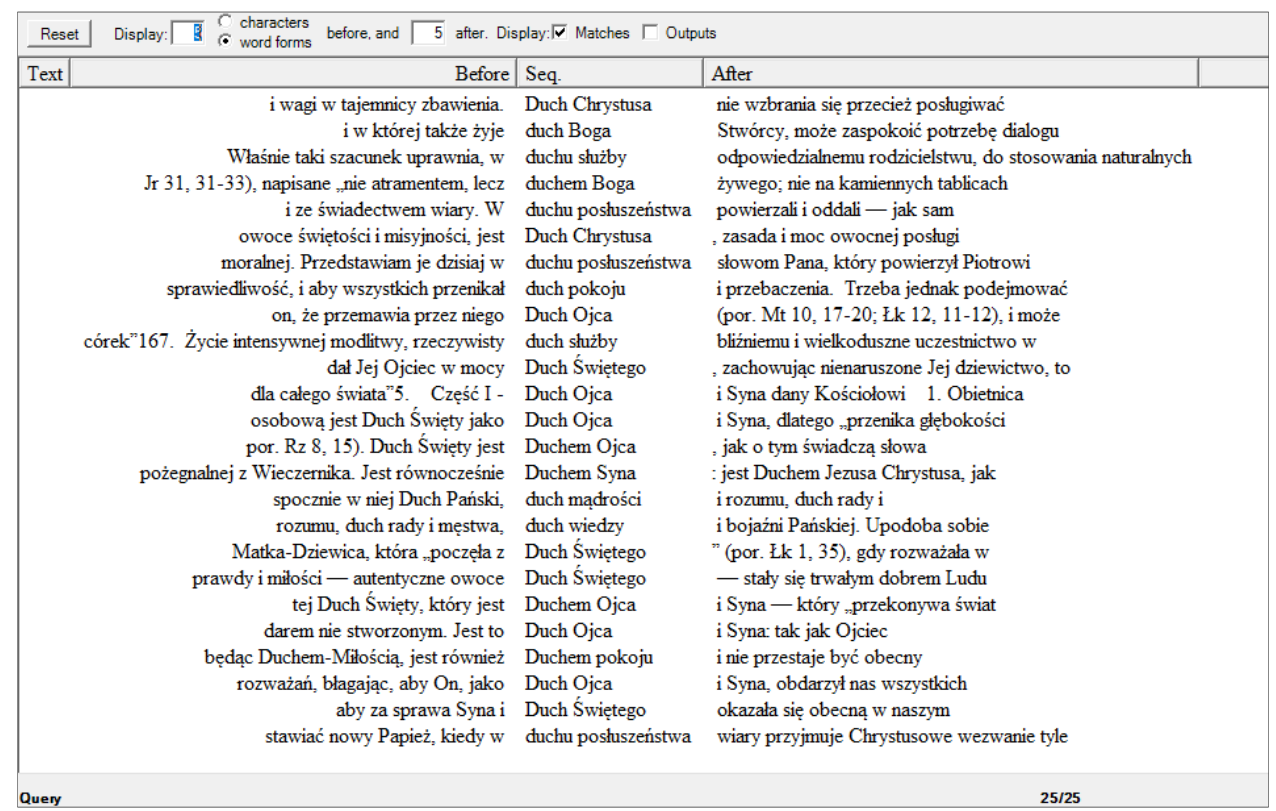

Figure 5 - Duch $\mathrm{N}_{\text {génitiff }}$

Par ailleurs il est à signaler que la présence du lexème duch dans la version polonaise n'implique pas forcément l'emploi du lexème esprit dans la version française, ce mot étant polysémique dans les deux langues. Tel est le cas de l'emploi $w$ duchu postuszeństwa (dans un esprit d'obéissance) où ces lexèmes ne désignent ni 'Personne Divine' ni 'nature spirituelle de l'homme' mais plutôt une référence à une valeur positive : 'au nom de'. Dans un passage d'Evangelium Vitce:

PL : Właśnie taki szacunek uprawnia, w duchu służby odpowiedzialnemu rodzicielstwu, do stosowania naturalnych metod regulacji płodności...

FR : C'est précisément cette attitude qui rend légitime, pour aider l'exercice de la responsabilité dans la procréation, le recours aux méthodes naturelles de régulation de la fertilité...

cette référence est envisagée comme une finalité et exprimée par la préposition pour.

Nous avons systématiquement examiné les suites $\mathrm{N}$ Adj avec accord en cas afin de repérer un certain nombre de collocations standard comme : 
Bon : sposób godny (une façon digne)

Ver : cecha characterystyczna (un trait caractéristique)

AntiVer + AntiBon : forma błędna (une forme erronée)

Remarquons, cependant, que les suites $\mathrm{N}$ Adj les plus récurrentes correspondent à des locutions, surtout à des quasi-locutions (dans la terminologie de la Lexicologie Explicative et Combinatoire, cf. Mel'čuk, 2013), comme czase ostateczne (les derniers temps), istota ludzka (un être humain), obraz Boży (l'image de Dieu) ou à des noms propres, comme Syn Boży (Fils de Dieu), Piśmo Święte (La Sainte Écriture).

Relevons aussi que les suites N Adj avec l'adjectif mélioratif prawdziwy (authentique, véritable) sont assez fréquentes. On trouve un total de 89 occurrences, entre autres: prawdziwy sens (le sens authentique), prawdziwe znaczenie (le sens réél), prawdziwy Bóg ou Bóg prawdziwy (le véritable Dieu), sposób prawdziwy ou prawdziwy sposób (une manière véridique).

Terminons en signalant que, dans la version française des Encycliques, l'adjectif polonais prawdziwy est traduit par différents adjectifs français (authentique, véritable, réel, véridique...) en fonction de sa combinatoire avec le $\mathrm{N}$.

\section{CONCLUSION}

Nous avons fait un certain nombre de remarques ayant un caractère lexicométrique, lexicologique et syntactico-sémantique concernant les Encycliques de Jean-Paul II. D'une part, nous avons appliqué une méthodologie d'analyse linguistique. D'autre part, nous avons voulu montrer comment la gestion de corpus avec une plate-forme d'ingénierie linguistique comme NooJ a l'avantage de pouvoir disposer de connaissances linguistiques stockées sous forme de dictionnaires électroniques. On peut ainsi aller bien au delà du traitement offert par des logiciels qui ne proposent que des anamorphoses des corpus introduits, lesquelles n'apportent pas de données nouvelles au sens strict de la théorie de l'information, puisque l'on ne peut obtenir que des restructurations de l'input textuel. De surcroît, NooJ permet de créer et de partager des ressources linguistiques élaborées pendant l'analyse d'un corpus donné.

Les observations que nous avons faites ci-dessus ne constituent qu'une toute première approximation ${ }^{9}$, bien imprécise, au corpus analysé et n'ont

\footnotetext{
${ }^{9}$ Pour une analyse lexicologique beaucoup plus fine concernant le texte des Encycliques, cf., par exemple, Śliwa à propos des emprunts polonais et français du mot fundamentum.
} 
pour but que de montrer comment on pourrait entreprendre la construction de grammaires locales discursives des principales notions contenues dans ces textes. Une grammaire locale (qui peut rendre compte aussi bien des relations prédicatives que des relations collocationnelles) peut être conçue comme un transducteur modélisant la cooccurrence syntactico-sémantique (prédicats appropriés) et la cooccurrence lexicale restreinte (collocations) ${ }^{10}$ pour une notion donnée, représentée par un verbe ou par un nom (plus rarement par un adjectif). Le fait d'élaborer des grammaires locales concernant le discours d'un auteur à partir d'un corpus permet de faire émerger les particularités de son modus scribendi.

Il n'y a pas de doute que ce type de recherche pourrait s'avérer fructueuse et qu'elle pourrait, notamment, constituer un excellent sujet pour une ou plusieurs thèses de doctorat, que nous appelons de nos vœux.

\section{BIBLIOGRAPHIE}

Blanco, Xavier. « Remarques sur la variation diachronique des collocations ». Cahiers de Lexicologie, vol. 116, 2020, pp. 71-94.

Gross, Gaston. Manuel d'analyse linguistique. Presses Universitaires du Septentrion, 2012.

Gross, Maurice. « Une grammaire locale de l'expression des sentiments ». Langue française, vol. 105, 1995, pp. 70-87.

Mel’čuk, Igor, et Alain Polguère. Lexique actif du français. De Bœck Supérieur, 2007.

Mel'čuk, Igor. «Collocations dans le dictionnaire ». Les écarts culturels dans les dictionnaires bilingues, dir. Thomas Szende (éd.), Honoré Champion, 2003, pp. 19-64.

Mel'čuk, Igor. " Tout ce que nous voulions savoir sur les phrasèmes, mais... » Cahiers de lexicologie, vol. 102, 2013, pp. 129-149.

Silberztein, Max. La formalisation des langues. L'approche de Nooj. ISTE Éditions, 2015.

Śliwa, Dorota. "Le mot fundamentum et ses relations en discours polonais et en français ». En quete de sens. Études dédiées à Marcela Świątkowska - W poszukiwaniu znaczeń. Studia dedykowane Marceli Świątkowskiej, dir. Joanna Górnikiewicz, Halina Grzmil-Tylutki et Iwona Piechnik, Wydawnictwo UJ, 2010, pp. 580-593.

\footnotetext{
${ }^{10}$ Nous avons déjà fait remarquer qu'il n'est pas toujours simple de décider si, au sein d'un syntagme donné (de type $\mathrm{N}$ Adj, par exemple), nous avons une sélection de type sémantique prédicat-argument $(\operatorname{Adj} \rightarrow \mathrm{N})$ ou une sélection de type lexical collocateur-collocatif $(\mathrm{N} \rightarrow \mathrm{Adj})$. Il existe certainement un continuum entre ces deux types de sélection qui ne peut être étudié de façon tout à fait convenable qu'en introduisant une perspective diachronique (Blanco, à paraître).
} 


\section{ÉLÉMENTS POUR UNE ANALYSE LEXICOLOGIQUE DES ENCYCLIQUES DE JEAN-PAUL II}

\section{Ré s u mé}

L'article propose quelques exemples d'analyse lexicologique et syntactico-sémantique, à l'aide du logiciel d'ingénierie linguistique NooJ 5.0, appliquée au corpus des quatorze encycliques de Jean-Paul II. Après une brève introduction, la première section de l'article $(\S 1)$ concerne l'aspect lexicométrique du corpus. La deuxième section $(\$ 2)$ aborde l'analyse syntactico-sémantique et la mise en évidence des structures prédicat-argument. La troisième section $(\S 3)$ présente quelques éléments visant l'étude des collocations (combinatoire lexicale restreinte). La quatrième et dernière section $(\S 4)$ propose un début d'analyse contrastive français-polonais.

Mots clés : schéma d'arguments ; collocation ; grammaire locale ; fonction lexicale ; plate-forme NooJ.

\section{ELEMENTY LEKSYKOLOGICZNEJ ANALIZY ENCYKLIK JANA PAWŁA II}

\section{Streszczenie}

W niniejszym artykule proponujemy kilka przykładów analizy leksykologicznej i składniowosemantycznej, zrealizowanej za pomocą oprogramowania inżynierii lingwistycznej NooJ 5.0 i zastosowanej do korpusu czternastu encyklik Jana Pawła II. Po krótkim wprowadzeniu w pierwszej części artykułu (§ 1) badamy aspekt leksykometryczny korpusu. Kolejna część (§ 2) dotyczy analizy składniowo-semantycznej i wyróżniania struktur predykatowo-argumentowych. Część trzecia (§ 3) przedstawia niektóre elementy opisania kolokacji (ograniczona kombinatoryka leksykalna). Czwarta i ostatnia część (§ 4) zawiera zalążek francusko-polskiej analizy kontrastywnej.

Slowa kluczowe: struktura argumentowo-predykatowa; kolokacja; gramatyka lokalna; funkcja leksykalna; platforma NooJ. 